\title{
A novel polymorphism, E254K, in the 5-lipoxygenase gene associated with bronchial asthma
}

\author{
CHUNYING BAI, EIKO MATSUI, HIDENORI OHNISHI, KAORI KIMATA, KIMIKO KASAHARA, \\ HIDEO KANEKO, ZENICHIRO KATO, TOSHIYUKI FUKAO and NAOMI KONDO
}

Department of Pediatrics, Graduate School of Medicine, Gifu University, Gifu, Japan

Received September 24, 2007; Accepted November 13, 2007

\begin{abstract}
Cysteinyl-leukotrienes are important proinflammatory mediators in bronchial asthma (BA) and are derived from arachidonic acid by the action of 5-lipoxygenase. We identified a novel polymorphism, c.760 G>A $(\mathrm{E} 254 \mathrm{~K})$, in exon 6 of the 5-lipoxygenase gene (5-LO). This substitution was detected in 11 out of 180 patients with BA, but not in any of the 150 non-allergic subjects. The frequency of c.760 G>A showed a significant difference between BA and non-allergic subjects $(\mathrm{P}=0.0007)$. The c.760 $\mathrm{G}>\mathrm{A}$ polymorphism existed at the surface edge of the $\mathrm{C}$-terminal catalytic domain, and the E-to-K substitution changed the charge of the side chain from negative to positive. Thus, our results suggest that $\mathrm{E} 254 \mathrm{~K}$ in the 5-LO might be associated with BA.
\end{abstract}

\section{Introduction}

Bronchial asthma (BA) is a multifactorial genetic disease (1). Cysteinyl-leukotrienes (cys-LTs) play an important proinflammatory role in both early- and late-phase asthmatic responses (2). Cys-LTs constitute a class of potent biological mediators of inflammation and anaphylaxis. 5-lipoxygenase is an essential enzyme which catalyzes the first committed steps in the biosynthetic pathway leading to the production of cys-LTs (3-6). The actions of 5-lipoxygenase result in the

Correspondence to: Eiko Matsui, Department of Pediatrics, Graduate School of Medicine, Gifu University, 1-1 Yanagido, Gifu 501-1193, Japan

E-mail: eikom@gifu-u.ac.jp

Abbreviations: SNP, single-nucleotide polymorphism; 5-LO, 5lipoxygenase gene; FLAP, 5-lipoxygenase-activating protein; BA, bronchial asthma; cys-LTs, cysteinyl-leukotrienes; PBMCs, peripheral blood mononuclear cells; EIA, enzyme immunoassay; ELISA, enzyme-linked immunosorbent assay; 5-HPETE, 5-hydroperoxyeicosatetraenoic acid; ICM, Internal Coordinate Mechanics; GAPDH, glyceraldehyde-3-phosphate dehydrogenase; c., cDNA

Key words: single-nucleotide polymorphism, 5-lipoxygenase gene, bronchial asthma, leukotrienes sequential conversion of arachidonic acid to 5-hydroperoxyeicosatetraenoic acid (5-HPETE) and then to leukotriene (LT) A4.

The 5-LO is located on chromosome 10q11.2 (7). In several studies, the addition of an Sp-1 binding motif (-GGG CGG-) or the deletion of one or two $\mathrm{Sp}-1$ binding motifs in the 5-LO core promoter, has been associated with reduced gene expression $(8,9)$. In addition, the 5-lipoxygenaseactivating protein (FLAP) promoter gene polymorphisms (21A repeat and $18 \mathrm{~A}$ repeat) were reported to be associated with BA (10). Recent evidence demonstrated that upregulation of 5-LO and FLAP mRNAs might be involved in the increased leukotriene synthesis and play an important role in the pathogenesis of BA (11).

In this study we identified single-nucleotide polymorphisms (SNPs) in the 5-LO and researched the relationship between SNPs in the 5-LO and BA.

\section{Materials and methods}

Patients and non-allergic subjects. One hundred and eighty BA patients (105 males and 75 females, mean age 8.4 47.6 years of age) and 150 non-allergic subjects (90 males and 60 females, mean age 9.5 \pm 8.2 years of age) were studied. The diagnosis of BA was made according to the criteria of the American Thoracic Society. The non-allergic subjects were healthy and did not have a history of allergic diseases. All of the subjects were randomly selected from patients attending our hospital. Informed consent was obtained from all individuals or from their parents.

Detection of SNPs in 5-LO. Neutrophils were collected from heparinized blood. Genomic DNA was extracted from neutrophils with a Sepagene kit (Sanko Junyaku, Tokyo, Japan). The fourteen exons of 5- $L O$ were amplified using the PCR technique and sequenced using an ABI 3100 DNA autosequencer (Applied Biosystems, CA) in certain individuals with BA $(n=16)$ and certain non-allergic subjects $(n=14)$. For further study, the E254K substitution was detected in all individuals with BA $(\mathrm{n}=180)$ and non-allergic subjects $(\mathrm{n}=150)$, and the other three silent polymorphisms (c.21 $\mathrm{C}>\mathrm{T}$, c. $270 \mathrm{G}>\mathrm{A}$, c. $1728 \mathrm{~A}>\mathrm{G}$ ) were detected in 60 individuals with $\mathrm{BA}$ and 60 non-allergic subjects. The primer details for the PCR used in the detection of these polymorphisms are shown in Table I. 
Table I. Primer details for PCR used to detect 4 polymorphisms.

\begin{tabular}{|c|c|c|c|}
\hline Primer & Sequence & Amplified product size & Annealing temp $\left({ }^{\circ} \mathrm{C}\right)$ \\
\hline c. $21 \mathrm{C}>\mathrm{T}$ FP & 5'CGCCATGCCCTCCTACAC3' & $150 \mathrm{bp}$ & 56 \\
\hline c. $21 \mathrm{C}>\mathrm{T}$ RP & 5'CCACGCTCGAAGTCGTTGTA3' & & \\
\hline c. $270 \mathrm{G}>\mathrm{A}$ FP & 5'GTGCCACAGCAGCATACCT3' & 401 bp & 55 \\
\hline c. $270 \mathrm{G}>\mathrm{A} \mathrm{RP}$ & 5'CCTGCACAGCAGTGTCATTC3' & & \\
\hline c.760 G>A (E254K) FP & 5'CCTGGTAGAGCGGGTCATGAATC3' & 179 bp & 62 \\
\hline c.760 G>A (E254K) RP & 5'ACCTCCTGCTCCAAGGGGAGCT3' & & \\
\hline c. 1728 A>G FP & 5'GAAAGAGGATGGACGGACTG3' & 295 bp & 55 \\
\hline c.1728 A>G RP & 5'CTCGTTTTCCTGGAACTGGC3' & & \\
\hline
\end{tabular}

FP, forward primer; RP, reverse primer.

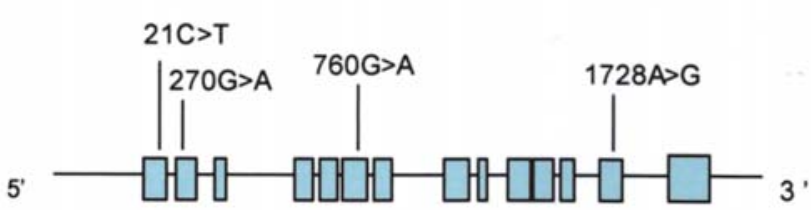

Figure 1. Gene structure and polymorphisms investigated in the 5-LO. The positions marked were found in polymorphisms in the Japanese population.

Measurement of urinary LTE4. Urine samples from 14 nonallergic subjects and 16 individuals with BA (13 without E254K and 3 with E254K), were stored at $-80^{\circ} \mathrm{C}$, and analyzed within 1 month of collection. The urinary creatinine level was determined by a creatinine test kit (Pure Auto S CRE-L, Daiichi-kagaku, Tokyo, Japan). Urinary LTE4 concentrations determined by EIA were corrected for recovery of $\left[{ }^{3} \mathrm{H}\right]$-LTE4. The urinary LTE4 level was expressed as $\mathrm{pg} / \mathrm{mg}$ of creatinine.

Measurement of LTB4 production from neutrophils. Neutrophils were collected from heparinized blood in 14 non-allergic subjects and 16 individuals with BA (13 without E254K and 3 with E254K), and $2 \times 10^{6}$ cells $/ \mathrm{ml}$ were cultured in an RPMI-1640 medium with $15 \%$ fetal calf serum, after stimulation with $1 \mu \mathrm{M}$ ionomycin. At 0,15 and 30 min after adding ionomycin, we aspirated $1 \mathrm{ml}$ of the culture medium and stored it at $-80^{\circ} \mathrm{C}$ for the measurement of LTB4. We used the LTB4 Immunoassay (R\&D Systems Inc., Minneapolis, $\mathrm{MN}$ ) to quantify the LTB4 concentration in a supernatant from the cultured ionomycin-stimulation neutrophils (12). The samples were applied to the C18 reverse-phase column, and were measured by ELISA.

Relative expression of 5-lipoxygenase mRNA. PBMCs were isolated from the heparinized blood of the 14 non-allergic subjects and 16 individuals with BA (13 without E254K and 3 with E254K) by gradient centrifugation in Ficoll-Paque (Pharmacia, Uppsala, Sweden) and stored at $-80^{\circ} \mathrm{C}$ for the extraction of mRNA. We quantified the relative expression of 5-lipoxygenase mRNA by real-time PCR. The real-time
Wild-type
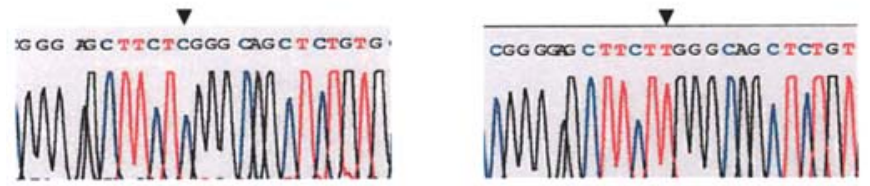

Figure 2. Big Dye terminator DNA sequence data of E254K in the reverse chain of 5-LO. The data of a patient with E254 (wild-type) are shown on the left and the data of a patient with K254 (mutant-homozygous type) are shown on the right.

PCR was carried out with a Light Cycler instrument (Roche, Mannheim, Germany) by using the Light Cycler SYBRGreen I RNA Master Kit (Roche). Each PCR cycle included denaturation at $95^{\circ} \mathrm{C}$ for $10 \mathrm{sec}$, primers annealing at $57^{\circ} \mathrm{C}$ for $10 \mathrm{sec}$, and a final extension at $72^{\circ} \mathrm{C}$ for $8 \mathrm{sec}$. The cDNA was amplified using the following primers: sense primer 5 'actgg aaacacggcaaaaac 3 ' in exon 3 and anti-sense primer 5 'tcac ggggtaaatccttgtg3' in exon 4 . The size of the PCR product was $96 \mathrm{bp}$ and the intron size between these two primers was $>26 \mathrm{~kb}$ (7). Glyceraldehyde-3-phosphate dehydrogenase (GAPDH) was used as the internal control for real-time quantitative PCR and we analyzed the relative expression of 5-lipoxygenase mRNA by the $2^{-\Delta \Delta C t}$ method (13).

Homology structural model of 5-lipoxygenase E254 and K254. An initial homology model structure of 5-lipoxygenase was made by a FUGUE (http://www-cryst.bioc.cam.ac.uk/ $\sim$ fugue/) server. Coral 8R-lipoxygenase (2fnq) was selected as the most homologous template of 5-lipoxygenase from the Protein Data Bank (www.rcsb.org/pdb). The target-template identity rate is $39 \%$. On the basis of this initial structural model, we optimized the structure and performed energy minimization using Internal Coordinate Mechanics (ICM) Pro version 3.3 (MolSoft). Furthermore, we generated the mutational form of this structure using the same software package. The validity of these structural models was evaluated by Ramachandran plot. The surface electrostatic potentials of these wild and mutant structures were calculated using Molmol software. 
Table II. Allele and genotype frequencies of 5- $L O$ silent SNPs.

\begin{tabular}{|c|c|c|c|}
\hline & Non-allergic subjects $(n=60)$ & Bronchial asthma patients $(n=60)$ & P-value \\
\hline \multicolumn{4}{|c|}{ Allele frequency } \\
\hline \multicolumn{4}{|c|}{ c. $21 \mathrm{C}>\mathrm{T}$} \\
\hline $\mathrm{C}$ & $108(0.9)$ & $110(0.917)$ & \\
\hline $\mathrm{T}$ & $12(0.1)$ & $10(0.083)$ & 0.824 \\
\hline \multicolumn{4}{|c|}{ c. $270 \mathrm{G}>\mathrm{A}$} \\
\hline $\mathrm{G}$ & $115(0.958)$ & $119(0.992)$ & \\
\hline A & $5(0.042)$ & $1(0.008)$ & 0.213 \\
\hline \multicolumn{4}{|c|}{ c. $1728 \mathrm{~A}>\mathrm{G}$} \\
\hline A & $113(0.942)$ & $116(0.967)$ & \\
\hline $\mathrm{G}$ & $7(0.058)$ & $4(0.033)$ & 0.539 \\
\hline \multicolumn{4}{|c|}{ Genotype frequency } \\
\hline \multicolumn{4}{|c|}{ c. $21 \mathrm{C}>\mathrm{T}$} \\
\hline $\mathrm{CC}$ & $48(0.8)$ & $52(0.867)$ & \\
\hline $\mathrm{CT}$ & $12(0.2)$ & $6(0.100)$ & 0.463 \\
\hline $\mathrm{TT}$ & 0 & $2(0.033)$ & \\
\hline \multicolumn{4}{|c|}{ c. $270 \mathrm{G}>\mathrm{A}$} \\
\hline GG & $55(0.917)$ & $59(0.983)$ & \\
\hline GA & $5(0.083)$ & $1(0.017)$ & 0.207 \\
\hline AA & 0 & 0 & \\
\hline \multicolumn{4}{|c|}{ c. $1728 \mathrm{~A}>\mathrm{G}$} \\
\hline AA & $53(0.883)$ & $56(0.933)$ & \\
\hline $\mathrm{AG}$ & $7(0.117)$ & $4(0.067)$ & 0.529 \\
\hline GG & 0 & 0 & \\
\hline
\end{tabular}

Table III. Allele and genotype frequencies of 5-LO missense SNP.

Non-allergic subjects $(n=150) \quad$ Bronchial asthma patients $(n=180) \quad P$-value

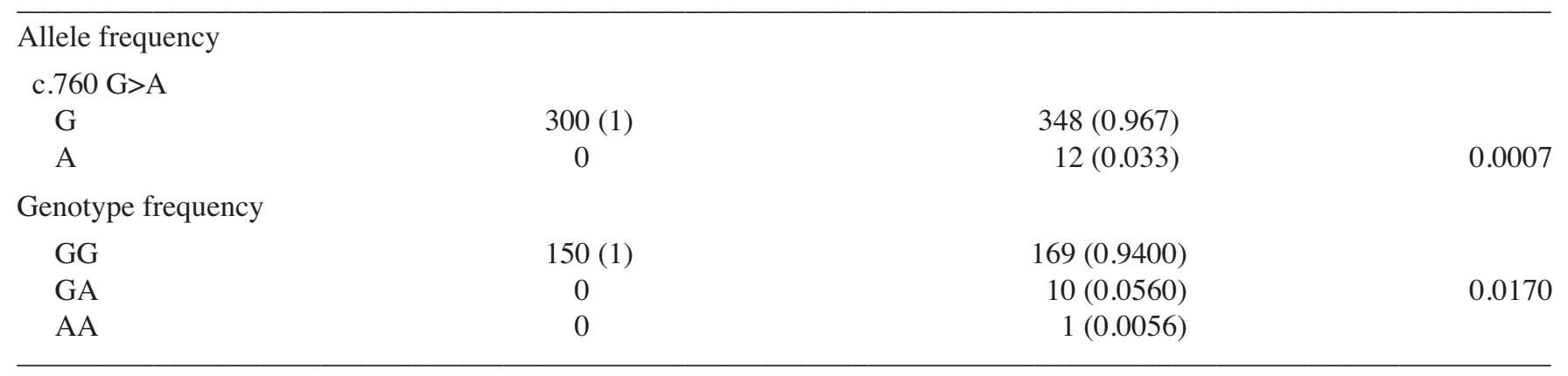

Statistical analyses. Allele and genotype frequencies were calculated for each locus and tested for Hardy-Weinberg equilibrium. Distribution of the genotype of E254K in the 5-LO was analyzed by Fisher's exact test. Probability $(\mathrm{P})$ values $<0.05$ were considered statistically significant. The significance of difference was analyzed by the two-sample t-test.

\section{Results}

Polymorphisms in the 5-LO. We identified 4 SNPs in the 5-LO in individuals with BA (Fig. 1). Three SNPs were silent polymorphisms: c.21 C>T (exon 1), c.270 G>A (exon 2) and c.1728 $\mathrm{A}>\mathrm{G}$ (exon 13). There were no differences in the frequencies of the three SNPs between individuals with BA and non-allergic subjects (Table II).

One SNP was a missense polymorphism c.760 G>A, and the amino acid at 254 changed from Glu (E) to Lys (K) (Fig. 2). We determined the prevalence of c.760 G>A $(\mathrm{E} 254 \mathrm{~K})$ in the $5-\mathrm{LO}$ of individuals with BA and non-allergic subjects. This SNP was found in $11(0.061)$ out of the 180 individuals with BA. One was homozygous AA and 10 were heterozygous GA ( 2 out of the 10 were brother and sister). The mutant allele frequency was 0.033 in 180 individuals with BA. However, the mutant allele could not be detected in any of the 150 non-allergic subjects (Table III). There was a significant difference in the $\mathrm{E} 254 \mathrm{~K}$ frequency between 
LTE4

(pg/mg Cre)

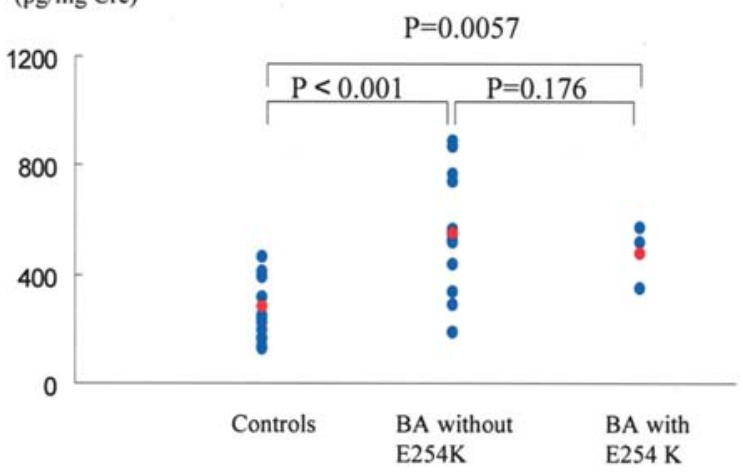

Figure 3. Urinary LTE4 production in individuals with bronchial asthma (BA) without E254K $(n=13)$ and with $\mathrm{E} 254 \mathrm{~K}(\mathrm{n}=3)$, and non-allergic subjects $(\mathrm{n}=14)$. The difference between individuals with bronchial asthma and non-allergic subjects was tested using a two-sample t-test. The urinary LTE4 productions were significantly higher in individuals with BA (without or with $\mathrm{E} 254 \mathrm{~K})$ than in non-allergic subjects $(\mathrm{P}<0.05)$. The red mark is the mean level.

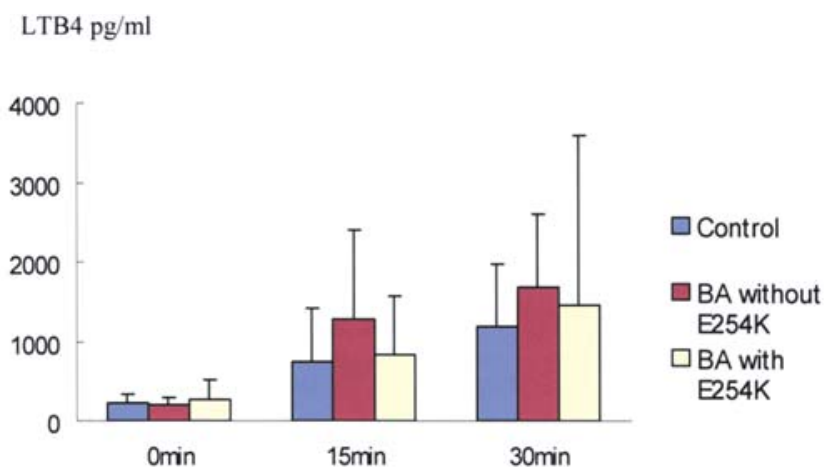

Figure 4. LTB4 production of ionomycin stimulation in neutrophils from individuals with bronchial asthma $(\mathrm{BA})$ without E254K $(\mathrm{n}=13)$ and with E254K $(n=3)$, and non-allergic subjects $(n=14)$. The significance of difference between groups of LTB4 was tested using a two-sample-t-test. We detected no difference in LTB4 production in resting neutrophils from individuals with BA compared to non-allergic subjects. The mean level of LTB4 showed a tendency to increase more in individuals with BA (without or with E254K) than in non-allergic subjects after stimulation by ionomycin at 15 and $30 \mathrm{~min}$, but there were no significant differences $(\mathrm{P}>0.05)$.

individuals with BA and non-allergic subjects (Fisher's exact test, $\mathrm{P}=0.0007)$.

Associations of E254K with urinary LTE4 or LTB4 production in neutrophils. To examine the functional effects of c.760 $\mathrm{G}>\mathrm{A}(\mathrm{E} 254 \mathrm{~K})$ in the $5-\mathrm{LO}$, we measured the urinary LTE4 levels in individuals with BA (without or with E254K) and non-allergic subjects. The urinary LTE4 levels were significantly higher in individuals with BA (without or with E254K) than in non-allergic subjects $(\mathrm{P}<0.05)$. However, the mean level of urinary LTE4 concentrations showed a tendency to decrease in individuals with BA and with E254K compared to those with BA but without E254K, although there was no significant difference (Fig. 3).

Furthermore, we measured the LTB4 concentrations in neutrophils isolated from individuals with BA (without or with E254K) and non-allergic subjects before and after
5-LO mRNA expression

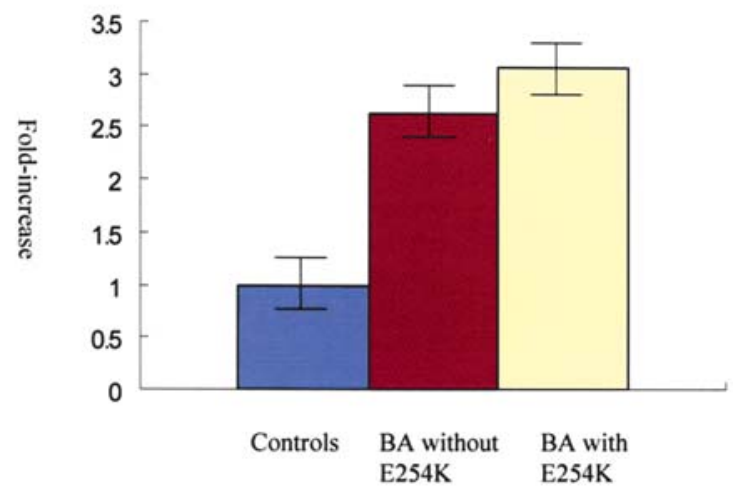

Figure 5. Relative expression of 5-lipoxygenase mRNA in individuals with bronchial asthma (BA) without E254K $(n=13)$ and with E254K $(n=3)$ and non-allergic subjects $(n=14)$. The $Y$-axis on the left indicates the foldincrease compared to the mRNA expression in non-allergic subjects shown by the open bars. The relative expression of 5-lipoxygenase mRNA in individuals with BA but without E254K increased 2.6-fold above that in non-allergic subjects; in individuals with BA and with E254K it increased 3.1-fold above that in non-allergic subjects.

stimulation by ionomycin. There were no differences in LTB4 production in resting neutrophils from individuals with BA compared to non-allergic subjects. The mean level of LTB4 concentration showed a tendency to increase in individuals with BA (without or with E254K) compared to non-allergic subjects after stimulation by ionomycin at 15 and $30 \mathrm{~min}$ (Fig. 4). The mean level of LTB4 concentrations in neutrophils showed a tendency to decrease in individuals with BA and with E254K compared to those with BA but without E254K, although there was no significant difference $(\mathrm{P}>0.05)$.

Relative expression of 5-lipoxygenase mRNA. We used the real time PCR (LightCycler 1.5 Instruments and SYBRGreen I system) to quantify the relative expression of 5 -lipoxygenase mRNA in individuals with BA (without or with E254K) and non-allergic subjects. GAPDH was used as the internal control for real-time quantitative PCR and the relative expression of 5-lipoxygenase mRNA was analyzed by the $2^{-\Delta \Delta C t}$ method. The relative expression of 5-lipoxygenase mRNA in individuals with BA but without E254K increased 2.6-fold above that in non-allergic subjects. In individuals with BA and with E254K the relative expression increased 3.1 -fold above that in non-allergic subjects. The relative expression of 5-lipoxygenase mRNA was higher in individuals with BA and with E254K than in those with BA but without E254K (Fig. 5).

Homology structural model of 5-lipoxygenase E254 and $K 254$. The human 5-lipoxygenase structural model consisted of the N-terminal $\beta$-barrel domain, thought to interact with lipids, and the C-terminal catalytic domain containing the active site that is the iron-binding site and the substratebinding cleft. We found that the SNP of 5-LO, E254K, existed at the surface edge of the $\mathrm{C}$-terminal catalytic domain, but this site was far from the active site of that enzyme (Fig. 6A). However, part of glutaminic acid 254 and lysine 254 had side chains, which obviously are exposed to 
A

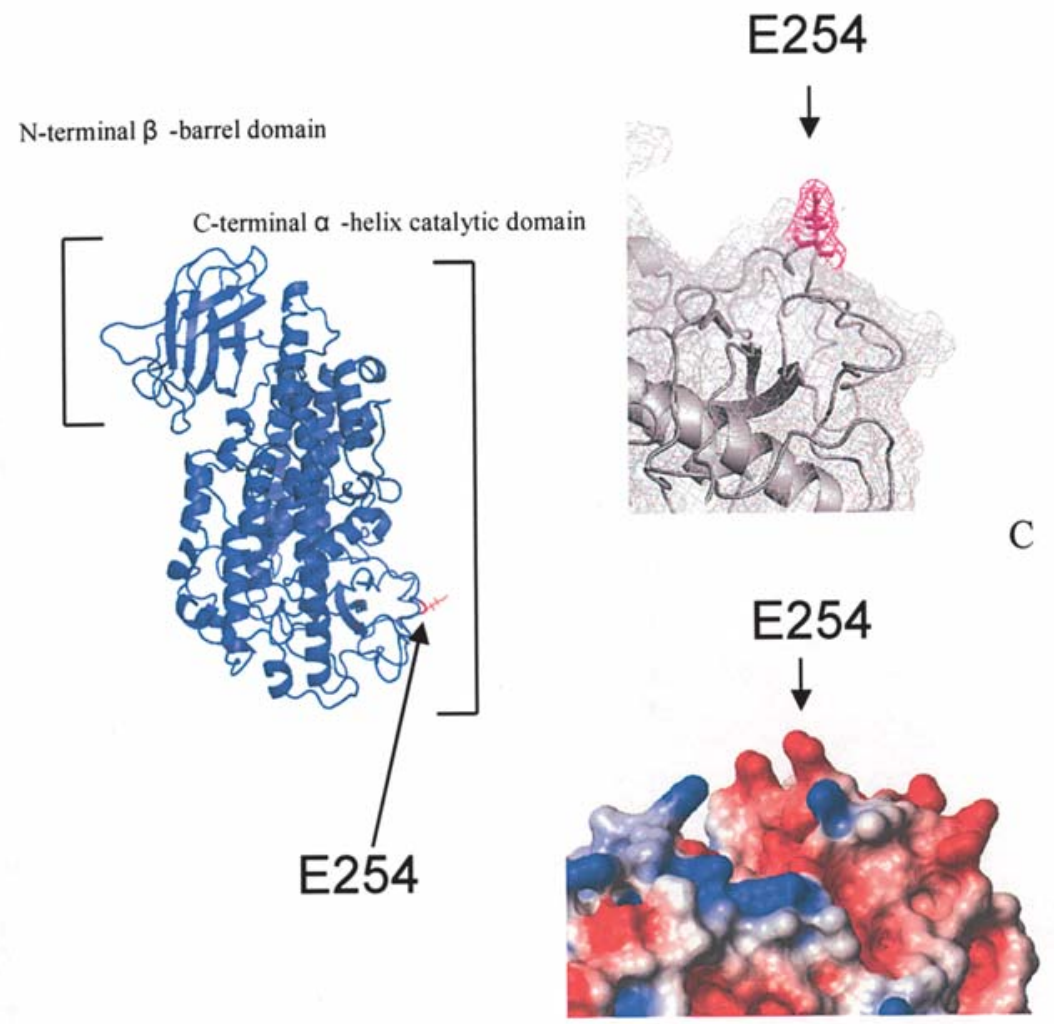

B
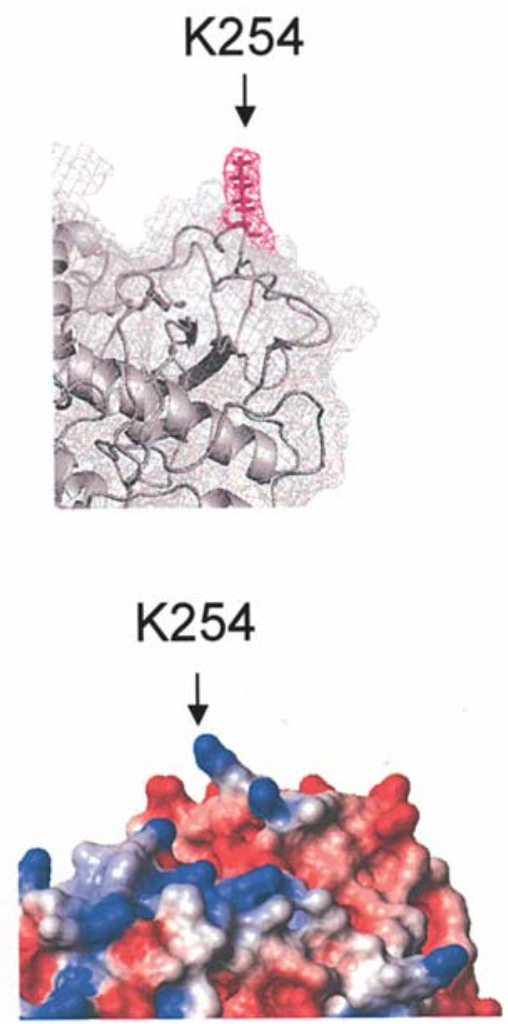

Figure 6. (A) Homology model structure of 5-lipoxygenase. The arrow indicates the part of the E254K polymorphism. (B) Comparison of the orientation of the side chains of glutamine acid 254 and lysine 254. (C) The focuses of the surface electrostatic potential of 5-lipoxygenase structures. Electrostatic potential representations of these two with red indicate areas of negative charge and blue indicate areas of positive charge. Arrows indicate the part of E254 and K254.

the solvent in these structural models (Fig. 6B), and the E-to$\mathrm{K}$ substitution changed the charge of the side chain from negative to positive (Fig. 6C).

\section{Discussion}

Clinically similar asthma symptoms may be caused by different mechanisms (14). Chronic airway inflammation is a feature of asthma. Recently, evidence has demonstrated that leukotriene C4, D4 and E4 increase in the serum, urine and exhaled breath condensate (EBC) of asthma patients $(11,15)$. The first committed enzyme in the biosynthetic pathway leading to the production of leukotrienes is 5-lipoxygenase. The addition of an Sp-1 binding motif (-GGGCGG-) or deletion of one or two Sp-1 binding motifs in the 5- $L O$ core promoter has been associated with reduced gene expression (9). We studied the polymorphisms in the 5-LO and attempted to clarify the relationship between the novel polymorphism (c.760 G>A) and bronchial asthma.

We found a missense SNP and three silent SNPs in the 5-LO. All patients who had an E254K substitution suffered from BA. There was a significant difference in the E254K frequency between individuals with $\mathrm{BA}$ and non-allergic subjects. Three other silent SNPs (c.21 C>T, c.270 G>A and c.1728 A>G) described previously (16), were also identified, but there were no significant differences in the frequencies between individuals with BA and non-allergic subjects.
To examine the functional effects of c.760 G>A (E254K) in the $5-L O$, we measured the production of urinary LTE4 and LTB4 in neutrophils in individuals with BA (without or with E254K) and non-allergic subjects. We found that urinary LTE4 production was significantly higher in individuals with BA (without or with E254K) than in nonallergic subjects. Furthermore, the mean level of LTB4 production in neutrophils showed a tendency to increase in individuals with BA (without or with E254K) more than in non-allergic subjects after stimulation by ionomycin. These results support the theory that leukotrienes play an important role in BA. The relative expression of 5-lipoxygenase mRNA in individuals with BA (without or with E254K) increased 2.6- or 3.1-fold above that in non-allergic subjects. This result supports the opinion of Koshino et al that the upregulation of 5-lipoxygenase mRNA might be involved in the increased leukotriene synthesis and play an important role in the pathogenesis of asthma (11). In this study, the relative expression of 5-lipoxygenase mRNA was higher in individuals with BA and with E254K than in those with BA but without E254K. As a result, the 5-LO pathway productions (urinary LTE4 and LTB4 levels in neutrophils) should be higher in individuals with BA and with E254K than in those with BA but without E254K. However, in this study, the urinary LTE4 and LTB4 levels in neutrophils showed a tendency to decrease in individuals with BA and with E254K compared to those with BA but without E254K. This result may be 
caused by the change of the E-to-K substitution at amino acid 254. This SNP in the 5-LO, which changes the charge from negative to positive, may affect the stability of the 5-lipoxygenase. Therefore, this SNP is induced to inhibit the synthesis of cys-LTs.

In order to clarify the functional effect of E254K, we analyzed the structural model of 5-lipoxygenase. The human 5-lipoxygenase structural model consisted of the $\mathrm{N}$-terminal B-barrel domain, thought to interact with lipids, and the Cterminal catalytic domain containing the active site that is the iron-binding site and the substrate-binding cleft. Our new finding is that the substitution of $5-L O, \mathrm{E} 254 \mathrm{~K}$, existed at the surface edge of the C-terminal catalytic domain, but this site was far from the active site of that enzyme. However, part of glutamine acid 254 and lysine 254 had side chains, which are obviously exposed to the solvent in these structural models. Also, the E-to-K substitution changed the charge of the side chain from negative to positive, and it has been reported that this type of change can induce certain diseases $(17,18)$. A previous report showed that some of the other cellular proteins interact with 5-lipoxygenase using the yeast twohybrid screening method (19). Glutamine acid 254 might influence 5-lipoxygenase to interact with some other cellular proteins but not with FLAP or with the substrate of this enzyme (20-25). Pharmacogenetics is the study of how genetic differences influence the variability in patients' responses to therapy (26). Further studies may be necessary to define the relationship between these 4 SNPs and patients' response to therapy.

In conclusion, our study suggested that the c.760 G>A polymorphism, E254K, in the 5-lipoxygenase gene, is associated with bronchial asthma, and our findings can contribute to the evaluation of one of the genetic risk factors for this disease.

\section{Acknowledgements}

This study was supported by Health and Labour Science Research Grants for Research on Allergic Disease and Immunology from Ministry of Health, Labour and Welfare.

\section{References}

1. Bochner BS and Busse WW: Allergy and asthma. J Allergy Clin Immunol 115: 953-959, 2005.

2. Sanak M: Genetic variance of 5-lipoxygenase metabolic pathway in bronchial asthma. Int Rev Asthma 4: 70-80, 2002.

3. Chen XS, Sheller JR, Johnson EN and Funk CD: Role of leukotrienes revealed by targeted disruption of the 5-lipoxygenase gene. Nature 372: 179-182, 1994.

4. Samuelsson B: Leukotrienes: mediators of immediate hypersensitivity reactions and inflammation. Science 220: 568-575, 1983.

5. Samuelsson B, Dahlen SE, Lindgren JA, Rouzer CA and Serhan CN: Leukotrienes and lipoxins: structures, biosynthesis, and biological effects. Science 1237: 1171-1176, 1987.
6. Lewis RA, Austen KF and Soberman RJ: Leukotrienes and other products of the 5-lipoxygenase pathway. Biochemistry and relation to pathobiology in human diseases. $\mathrm{N}$ Engl $\mathrm{J}$ Med 323: 645-655, 1990

7. Funk CD, Hoshiko S, Matsumoto T, Radmark O and Samuelsson B: Characterization of the human 5-lipoxygenase gene. Proc Natl Acad Sci USA 86: 2587-2591, 1989.

8. Drazen JM and Silverman ES: Genetic determinants of 5-lipoxygenase transcription. Int Arch Allergy Immunol 118: 275-278, 1999.

9. Silverman ES and Drazen JM: Genetic variations in the 5lipoxygenase core promoter. Am J Respir Crit Care Med 161: $77-80,2000$.

10. Koshino T, Takano S, Kitani S, et al: Novel polymorphism of the 5-lipoxygenase activating protein (FLAP) promoter gene associated with asthma. Mol Cell Biol Res Commun 2: 32-35, 1999.

11. Koshino T, Takano S, Houjo T, et al: Expression of 5-lipoxygenase (5-LO) and 5-lipoxygenase-activating protein (FLAP) mRNAs in the peripheral blood leukocytes of asthma. Biochem Biophys Res Commun 247: 510-513, 1998.

12. Helgadottir A, Manolescu A, Thorleifsson G, et al: The gene encoding 5-lipoxygenase activating protein confers risk of myocardial infarction and stroke. Nat Genet 36: 233-239, 2004.

13. Livak KJ and Schmittgen TD: Analysis of relative gene expression data using real time quantitative PCR and the $2^{-\triangle \Delta \mathrm{Ct}}$ method. Methods 25: 402-408, 2001.

14. Caterina RD and Zampolli A: From asthma to atherosclerosis 5-lipoxygenase, leukotrienes, and inflammation. N Engl J Med 350: 4-7, 2004 .

15. Shibata A, Katsunuma T, Tomikawa M, Tan A, Yuki K, Akashi K and Eto Y: Increased leukotriene E4 in the exhaled breath condensate of children with mild asthma. Chest 130: 1718-1722, 2006

16. In K-H, Silverman ES, Asano K, et al: Mutations in the human 5-lipoxygenase gene. Clin Rev Allergy Immunol 17: 59-69, 1999.

17. Yuen PH, Ryan EA, Devroe E and Wong PKY: A single Glu (62)-to-Lys (62) mutation in the Mos residues of the R7Delta447Gag-tMos protein causes the mutant virus to induce brain lesions. Oncogene 20: 692-703, 2001.

18. Berretta F, Butler RH, Diaz G, et al: Detailed analysis of the effects of Glu/Lys $B 69$ human leukocyte antigen-DP polymorphism on peptide-binding specificity. Tissue Antigens 62: 459-471, 2003.

19. Provost P, Samuelsson B and Radmark O: Interaction of 5-lipoxygenase with cellular proteins. Biochemistry 96: 1881-1885, 1999.

20. Manev $\mathrm{H}$ and Tolga UZ: Primary cultures of rat cerebellar granule cells as a model to study neuronal 5-lipoxygenase and FLAP gene expression. Ann NY Acad Sci 890: 183-190, 1999.

21. Zimmer JS, Dyckes DF, Bernlohr DA and Murphy RC: Fatty acid binding proteins stabilize leukotriene A4: competition with arachidonic acid but not other lipoxygenase products. J Lipid Res 45: 2138-2144, 2004.

22. Voelkel NF, Tuder RM, Wade K, et al: Inhibition of 5-lipoxygenase-activating protein (FLAP) reduces pulmonary vascular reactivity and pulmonary hypertension in hypoxic rats. J Clin Invest 97: 2491-2498, 1996.

23. Lepley RA, Muskardin DT and Fitzpatrick FA: Tyrosine kinase activity modulates catalysis and translocation of cellular 5-lipoxygenase. J Biol Chem 271: 6179-6184, 1996.

24. Abramovitz M, Wong E, Cox ME, Richardson CD, Li C and Vickers PJ: 5-Lipoxygenase-activating protein stimulates the utilization of arachidonic acid by 5-lipoxygenase. Eur J Biochem 215: 105-111, 1993.

25. In KH, Asano K, Beier D, et al: Naturally occurring mutations in the human 5-lipoxygenase gene promoter that modify transcription factor binding and reporter gene transcription. J Clin Invest 99: 1130-1137, 1997.

26. Israel E: Genetics and the variability of treatment response in asthma. J Allergy Clin Immunol 115: 532-538, 2005. 